\title{
Estrategias de gestión en cuidadores de menores de 14 años en diálisis
}

\section{Management strategies for caregivers of under 14 -year dialysis patients}

\author{
Claudia Andrea Ramírez Perdomo' , Karol Lucía Vargas Acosta², Laura Catalina Ñañez Vargas ${ }^{3}$, Lina \\ María Duque Ramírez ${ }^{4}$
}

\section{Resumen}

Objetivo: Determinar las estrategias de gestión en el proceso de duelo de los cuidadores de menores de 14 años en diálisis en la unidad renal del Hospital Universitario Hernando Moncaleano Perdomo (HUHMP) de Neiva.

Materiales y métodos: Estudio de enfoque cualitativo, tipo fenomenológico; se empleó una entrevista semiestructurada. Los medios utilizados por las cuidadoras para sobrellevar el proceso de duelo según la teoría del Duelo Disfuncional de Gaskill y colaboradores, fueron estrategias de gestión internas eficaces: espiritualidad, motivación y resignación; estrategias de gestión internas ineficaces: culpa y sobreprotección; estrategias de gestión externas eficaces: apoyo brindado por la pareja, familia, personal de salud y apoyo social; estrategias de gestión externas ineficaces: apoyo ineficaz de pareja, familia, entorno social y del personal de salud. Encontrándose marcada la impotencia. Conclusiones: Las cuidadoras no aceptan su realidad, la resignación ayuda a sobrellevarla, por el contrario la impotencia lo impide. La resignación, que contribuye a disminuir los sentimientos de impotencia, se alcanza mediante las estrategias de gestión eficaces e ineficaces, aunque éstas últimas producen disconfort y desencadenan sentimientos de impotencia, limitando la realización de estrategias de gestión eficaces. Estos hallazgos amplían la visión del equipo de salud, especialmente en enfermería permitiendo reconocer que hay un cuidador que necesita ser cuidado.

Palabras clave: Cuidador, duelo, diálisis (Bireme, MeSH).

\section{Abstract}

Objective: To determine the management strategies in the grieving process of caregivers of under-14-year dialysis patients in renal unit at University Hospital Hernando Moncaleano Perdomo (HUHMP) in Neiva.

Materials and methods: A qualitative phenomenological study. A semi-structured interview was used. The means used by caregivers to cope with the grieving process according to the theory of Dysfunctional Grief by Gaskill et al., were effective internal management strategies: spirituality, motivation and resignation; ineffective internal management strategies: guilt and over protection; effective external management strategies: support provided by the couple, family, health staff and social support; ineffective external management strategies: Ineffective support from partner, family, social environment and health staff. It was found remarkable power lessness.

Conclusions: Caregivers do not accept their reality, resignation helps to cope with it; on the contrary, power lessness impedes it. The resignation, which helps to decrease feeling sof power lessness, is achieved by means of effective and ineffective management strategies, although the latter produce discomfort and trigger feelings of power lessness, limiting the implementation of effective management strategies. These findings broaden the view of the health team, especially the nursing staff allow ingrecognizing that a caregiver needs to be cared.

Keywords: Caregiver, grief, dialysis.

\section{Introducción}

Cuando se afecta la salud de un menor es necesario el apoyo de un cuidador, generalmente son los padres quienes asumen dicho rol. La presencia del cuidador en el menor en tratamiento dialítico es esencial, éste le proporciona los complejos cuidados que por su corta edad y/o diferentes factores asociados no puede realizar. La alteración de la salud por aparición de una enfermedad devastadora los

1 Magíster en Enfermería, Universidad Nacional de Colombia. Estudiante Doctorado en Enfermería, Universidad de Antioquia. Docente Universidad Surcolombiana, Huila.Correo electrónico: clauram1@hotmail.com.

2 Enfermera, Universidad Surcolombiana.

3 Estudiante de Enfermería. Universidad Surcolombiana.

4 Enfermera, Universidad Surcolombiana.

Recibido: 18/12/13 - Revisado: 15/03/2014 - Aceptado: 23/8/2014 
hará depender del tratamiento dialítico y del cuidador, éste último también experimenta cambios que alteran su cotidianidad y ocasionan modificaciones sustanciales en su vida, llevándolos a realizar un proceso de duelo.

Según la teoría de enfermería del duelo disfuncional, propuesta por Gaskill y colaboradores, la perdida es una experiencia común en todos los individuos, para sobrellevar el proceso de duelo éstos se valen de diferentes medios denominados estrategias de gestión, pueden ser internas o externas y según el confort o disconfort que ocasionan, son consideradas como eficaces o ineficaces ${ }^{[1]}$. Está teoría abarca la compleja realidad de los cuidadores durante el proceso de duelo, y conoce los medios internos y externos que influyen en esta etapa.

En este orden de ideas, la investigación tuvo como objetivo determinar las estrategias de gestión en el proceso de duelo de los cuidadores de menores de 14 años sometidos a tratamiento dialítico en la unidad renal del HUHMP.

Por esta razón, conocer las estrategias de gestión que ayudan al cuidador en su proceso de duelo, permite que se potencialicen las estrategias de gestión eficaces y contribuye a disminuir aquellas que al ser ineficaces producen disconfort; las estrategias de gestión internas, comprenden aspectos personales que dependen de cada individuo, requieren ser abordadas por profesionales capacitados; mientras, que las estrategias de gestión externas, pueden ser proporcionadas por individuos de la comunidad, por lo tanto los miembros del equipo de salud deben ser los primeros en proporcionar una atención beneficiosa.

\section{Materiales y métodos}

Se realizó un estudio con enfoque cualitativo, fenomenológico, en cuidadores de menores de 14 años en tratamiento dialítico en la unidad renal del HUHMP en la ciudad de Neiva, los criterios de inclusión tenidos en cuenta fueron: ser mayores de edad y aceptación voluntaria de participar, entre los criterios de exclusión estaba padecer alguna enfermedad mental. Se contó con aprobación del comité de ética de la institución, fundamentado en los principios éticos de la investigación, la resolución 8430 de 1993 y la Ley 911 de 2004

La información se obtuvo mediante una entrevista semiestructurada; los datos fueron analizados en el software atlas ti, se codificó los aportes de cada cuidadora de la siguiente manera: P1 (cuidadora 1) y así sucesivamente con todas las participaciones. Se realizó una prueba piloto con un participante con características similares a la población de estudio.

Las cinco cuidadoras que participaron, se encontraban en edad adulta; el $60 \%$ estaban casadas, $20 \%$ viuda y $20 \%$ soltera. Los niños: $60 \%$ eran de sexo femenino, $40 \%$ sexo masculino; el $80 \%$ están en diálisis peritoneal y $20 \%$ en hemodiálisis.

\section{Resultados}

Los resultados se agruparon en dos categorías de análisis, la primera denominada estrategias de gestión, según Gaskill son los medios que pueden aumentar el confort o disconfort durante el proceso de duelo, divididos en 13 subcategorías, estas se agruparon en internas cuando las estrategias eran realizadas por el propio individuo y externas cuando venían de personas que le rodeaban. Así mismo, se agruparon en eficaces o ineficaces, es decir si aumentaban o disminuían el confort durante el proceso de duelo

Teniendo en cuenta lo anterior, son estrategias de gestión internas eficaces: espiritualidad, motivación y resignación; estrategias de gestión internas ineficaces: culpa y sobreprotección; estrategias de gestión externas eficaces: apoyo brindado por la pareja, familia, personal de salud y apoyo social, y finalmente estrategias de gestión externas ineficaces: apoyo ineficaz de pareja, familia, entorno social y del personal de salud. La segunda estrategia analizada fue la impotencia, considerada como emergente porque aunque no es una estrategia se gestión se encontró marcada en las cuidadoras (Tabla 1)

Espiritualidad: Pinto la define como una fuerza interna de la persona misma, que le permite tomar decisiones, hacer reflexiones, sortear problemas y motivarse internamente para continuar altiva en las distintas dificultades, como también en lo bello de la vida y así desarrollar sus potenciales. ${ }^{[2]}$

Tabla 1. Categorías de análisis.

\begin{tabular}{|c|c|c|c|}
\hline & \multirow{2}{*}{ Internas } & Eficaces & $\begin{array}{l}\text { Espiritualidad } \\
\text { Motivación } \\
\text { Resignación }\end{array}$ \\
\hline & & Ineficaces & $\begin{array}{l}\text { Culpa } \\
\text { Sobreprotección }\end{array}$ \\
\hline \multirow[t]{2}{*}{ Estrategias de gestión } & \multirow{2}{*}{ Externas } & Eficaces & $\begin{array}{l}\text { Apoyo brindado por pareja } \\
\text { Apoyo brindado por familia } \\
\text { Apoyo brindado por personal de salud } \\
\text { Apoyo Social }\end{array}$ \\
\hline & & Ineficaces & $\begin{array}{l}\text { Apoyo ineficaz de pareja } \\
\text { Apoyo ineficaz de familia } \\
\text { Apoyo ineficaz del entorno social } \\
\text { Apoyo ineficaz del personal de salud } \\
\text { Impotencia }\end{array}$ \\
\hline
\end{tabular}


Igualmente, Barrera propone que el bienestar espiritual es un sentido de armonía interna, que incluye la relación con el propio ser, los otros, el orden natural, Dios, un poder o fuerza superior ${ }^{[3]} \mathrm{Aquí}$ se observa la importancia de una relación filial con una entidad superior por medio de la vida espiritual significativa para los cuidadores, esto se observa en expresiones como: “...me fortalecí en la vida espiritual, para llenarme más de fuerza... "(P1)

Motivación: Santrock la plantea, como el conjunto de razones por las que las personas se comportan de la forma en que lo hacen. El comportamiento motivado es vigoroso, dirigido y sostenido. ${ }^{[4]}$ Esta surge del interior del individuo para hacer frente a los acontecimientos cotidianos, ayuda a mejorar el confort ante circunstancias que alteran su cotidianidad, como ante el duelo generado por una pérdida.

Así mismo para Herrera y cols., la motivación representa lo que originariamente determina que la persona inicie una acción, se dirija hacia un objetivo y persista en alcanzarlo. ${ }^{[5]}$ En las cuidadoras se evidencia que la mayor motivación para continuar con su rol, es el menor sujeto de cuidado, este es el motor que las mueve a luchar, puesto que sus necesidades, fortalecen el vínculo afectivo, expresada en afirmaciones como: "...yo digo, hay que luchar mientras mi hija este viva, por ella toca luchar..." (P5)

Resignación: Para Rodríguez y cols., es la percepción de la imposibilidad de cambiar algún aspecto de la situación- problema, con la consiguiente tienden a la inacción. ${ }^{[6]}$ El individuo puede llegar a adaptarse a las circunstancias, pero no logra total satisfacción de aquello que ha sido modificado, se evidencia en expresiones como "¿qué más se puede hacer?", "toca", "hacerse a la idea"; demostrando un inconformismo por los cambios generado en su vida por las circunstancias que propician el duelo, descrito en frases como: “...A nivel personal mi vida ha cambiado, tengo que ser fuerte y seguir porque ¿qué más se puede hacer? ... (P3)

Culpa: En ocasiones es generada por el sentimiento de haber realizado acciones $u$ omisiones en el pasado y se cree son las causantes del problema del individuo, como afirma Pérez "la culpa es la emoción de angustia que surge como consecuencia de la realización de actos (físicos, mentales, fantaseados u omitidos) que transgreden el sistema de normas y valores de la persona", ${ }^{77]}$ expresado así: "... Yo digo algo malo he hecho en esta vida para que ella esté pagando las consecuencias, siempre me he sentido culpable... "(P1)

Sobreprotección: Vásquez afirma que es el resultado de una inadecuada interpretación de la afectividad; caracterizada por tratar de evitar la mayor cantidad de riesgos y dificultades al hijo, limitando así sus posibilidades de desarrollo. ${ }^{[8]}$ La mujer como madre biológica busca protegerlos, pero su rol se tergiversa al adoptar conductas sobreprotectoras, especialmente si la persona cuidada, por su estado de salud demanda mayor atención, como por ejemplo: "...mi esposo dice que si fuera por mi hacia una burbuja y metía a mis hijos para que nada les hiciera daño..." (P1)

Apoyo de pareja: Según Estrada citado por Garrido, el término puede ser descrito como una unidad de personalidades interactuantes que forman un sistema de emociones y necesidades, entrelazadas entre sí, de la más profunda naturaleza, pero implica algo más que la simple suma de dos personalidades; es ante todo, una nueva entidad conformada por las características de dos elementos que crean una relación con una identidad propia. ${ }^{[9]}$ Entre dicha entidad surge la necesidad de compensar las exigencias del conyugue, para que quien ha asumido el rol de cuidador encuentre apoyo emocional, físico y material, que permite hacer frente a las adversidades, especialmente las producidas durante el proceso de duelo, esta se evidencia en expresiones: "Mi esposo me da fuerza siempre que esta y me dice: -no llore, no este biste, tenemos que salir adelante por ella"(P1)

Apoyo de familia: Según Lopes, la familia es un espacio de socialización primaria, solidaridad y protección socia ${ }^{[10]}$, al ser la fuente de contacto más próxima que el individuo tiene con su exterior, lo que ésta porte o suprima influirá significativamente en el proceder del mismo. El soporte familiar es un importante amortiguador para enfrentar las dificultades que se pueden presentar entre los miembros de una familia. ${ }^{[1]} \mathrm{El}$ proporcionar bienes materiales aun cuando esto genere sacrificios de quien los dona, demuestra afecto; esto se refleja en: “...Mi mamá y papá son pobres, pero nos han colaborado mucho, nos han sostenido con una comida diaria, la principal que es el almuerzo, mis hermanos que tienen sus hogares, me han colaborado con dinero... "(P4)

Apoyo del personal de salud: Aunque los familiares y amigos proporcionar una ayuda fundamental y valiosa, en ocasiones no es suficiente y resulta necesario otro tipo de soluciones, en concreto, recurrir a servicios o instituciones de salud, ${ }^{[12]}$ que permite al cuidador crear un vínculo afectivo que estimule su labor, bien sea ante el trato recibido durante la atención o brindar consejos que ayuden a sobrellevar su situación de cuidado, esta relación se percibe en: "...empezando por la parte humana, desde que he venido los médicos, las enfermeras, siempre tratan de colabor ar mucho, gracias a Dios, cuando se está en estas dificultades se ve la parte humana de la gente ... "(P1)

Apoyo del entorno social: Según Sarason y otros, este es definido como la existencia o disponibilidad de personas en quienes se puede confiar, personas que se muestran preocupadas por el individuo, lo valoran y le demuestran aprecio. ${ }^{[13]} \mathrm{Al}$ ser el hombre un ser social por naturaleza necesita de los demás para desarrollarse y ante situaciones de vulnerabilidad necesita una fuente de ayuda externa, ésta brinda el apoyo emocional, espiritual y material necesario para compensar las necesidades, estas estrategias se muestra en: "... mi mamá y en el barrio reconocen mi labor como cuidadora, hay gente que sabe cómo he sido todo el tiempo, como me ha tocado la vida de dur a y me admiran, dicen que soy una mujer luchador a, echada para adelante, que vivo pendiente del niño, inclusive los vigilantes de acá me lo han dicho... "(P4)

Apoyo inefectivo de pareja: Garrido Garduño y colaboradores, afirman que en una relación de pareja es importante analizar la convivencia diaria, donde tienen que aprender a negociar e incluso reñir, en efecto, no hay parejas que no tengan problemas, pero la diferencia radica en que una pareja exitosa tiene la habilidad para enfrentarlos. ${ }^{[14]} \mathrm{E} 1$ no asumir las dificultades o hacerlo de forma inadecuada, puede generar disfunción familiar que afecta negativamente el bienestar de sus miembros especialmente de quienes requieren apoyo. Esta estrategia se refleja en frases como: “...el papá apenas la vio asi la dejo, la abuelita por parte de él le daba cositas, él llego al punto de decir que las instituciones le suministraban todo, que no necesitaba nada, no era verdad, la familia por parte del papá no volvió a visitarla, no saben del estado de ella."(P2)

Apoyo inefectivo de familia: El cuidador y su familiar enfermo están inmersos en la situación con su propia familia, sus necesidades responden a la vivencia común de cronicidad, se pueden llegar a desarrollar conflictos en el núcleo familiar o familia extensa, recayendo el peso de todo esto sobre el cuidador. ${ }^{[12]}$ Se evidencia como algunos juicios, acciones u omisiones de los familiares generan en él 
un disconfort dificultando el proceso de duelo: "...Pues bien, como le digo hay unos que se preocupan otros que no, no preguntan ni nada..." (P3)

Apoyo inefectivo del personal de salud: Es responsabilidad del profesional de salud hacer ver a la familia las ventajas o desventajas de las soluciones generadas ante las dificultades presentadas en el proceso salud - enfermedad, así como facilitar información sobre los servicios y recursos que puede utilizar. ${ }^{[15]}$ En ocasiones no es así, puesto que la información brindada al cuidador para que tome decisiones, lleva percepciones subjetivas, desinterés, falta veracidad o son insuficientes, generando inconformidad, temores y mal entendidos que le afectaran negativamente; esto se demuestra en expresiones como: "...en ese tiempo habia un doctor muy amargado, nunca me dijo como era la diálisis ni que el niño iba a estar de por vida con eso, solo me decía que estaba muy maly que le pidiera al santo al que más fe le tuviera; ese doctor tenia una forma muy fea de hablar, muchas mamás nos quejamos... "(P4)

Apoyo inefectivo del entorno social: Goodwin y otros afirman que la simple presencia de soporte social por si solo ya parece ser bastante relevante. ${ }^{[16]}$ Por esta razón, la ausencia del apoyo social afecta negativamente a los cuidadores, tiene mayor impacto cuando las intervenciones realizadas por la comunidad le maltratan, aljuzgarle de manera indiscriminada, esto se evidencia en expresiones como: “...Hay vecinos que dicen, descuidaron el niño; uno se siente mal, porque ¿Cuándo se quiere un mal para un hijo? Un señor decía: -Ustedes descuidaron el niño, ustedes tal cosa..." (P3)

Impotencia: La NANDA la define como la percepción de que las propias acciones no variarán significativamente el resultado; percepción de falta de control sobre la situación actual o un acontecimiento inmediato. ${ }^{[17]}$ Relaciona a un estilo de vida de poca autoayuda, ${ }^{[19]}$ que caracteriza la aparición de la impotencia y la percepción de incapacidad para modificar situaciones que se alteran en el desempeño del rol, genera cambios negativos en su estilo de vida; ésta realidad aprecian en expresiones como: “...en un minuto cambio todo lo que yo hacia antes, porque yo trabajaba y trataba de ayudar en la casa..." (P1)

\section{Discusión}

Al analizar las estrategias de gestión en los cuidadores de menores de 14 años en diálisis se encuentra que las estrategias de gestión externas eficaces e ineficaces, y las estrategias de gestión internas eficaces llevan a la resignación. Aunque a esta se puedellegar de las formas mencionadas, para mayor beneficio es necesario optar por las estrategias eficaces, ya que estas causan confort y evitan la aparición de estrategias de gestión ineficaces que generan disconfort.

Andreu en sus hallazgos plantea que "la actitud del cuidador puede ir desde la resignación a una motivación extrema, que le lleve a ser muy meticuloso y a sentirse responsable de todo lo que ocurra, sobreprotegiendo al paciente".[18] Llevando así a estrategias internas ineficaces como la sobreprotección. Contrario a lo que se esperaría, puesto que se requiere que haya resignación y continúe como estrategia eficaz, si llega la sobreprotección ésta es catalogada como una estrategia ineficaz, que no genera armonía interior.

A su vez, las estrategias de gestión externas eficaces están intimamente relacionadas con la motivación, éstas animan al cuidador a realizar su rol, creando sentimientos positivos que lo ayudan a resignarse. Ante la influencia que ejercen las personas que rodean a un individuo, el apoyo recibido por parte del personal de salud y del entorno social, potencian su espiritualidad permitiendo comprender su situación desde una perspectiva que abre paso a la esperanza.

Las estrategias de gestión externas ineficaces generan en el cuidador sentimientos de impotencia, que hace proporcionar un cuidado sobreprotector al considerar que todo es nocivo para el menor, genera sentimientos de culpa al asociarlo con intervenciones perjudiciales y a facilita la reaparición de sentimientos de impotencia que aumentan el disconfort durante el proceso de duelo. Por esta razón, Blanco afirma que la habilidad de cuidado se encuentra gravemente afectada por los sentimientos de impotencia, la falta de reconocimiento y la angustia generada por las crisis de la enfermedad, afectándose la autoestima y la calidad de vida del cuidador. ${ }^{[19]}$

A relacionar las estrategias de gestión con la impotencia, se encuentra que las estrategias de gestión externas ineficaces y la culpa, conduce a la impotencia, que a su vez limitan la realización estrategias de gestión eficaces internas y externas. Esta limitación ocasiona un estancamiento en el proceso de duelo; contrario a cuando están fortalecidas, ya que le ayuda a resignarse más rápido y disminuye los sentimientos de impotencia.

Además, al afectarse la salud de un menor es necesario el apoyo constante del cuidador, rol asumido principalmente por los padres, Tong señala que los menores dependen de sus padres para el apoyo complejo, continuo e intensivo que su condición requiere. ${ }^{[20]}$ En este estudio, el apoyo fue proporcionado por las madres en etapa adulta, coincidiendo con Montalvo quien encontró que en mayor proporción las mujeres desempeñan este rol ${ }^{[21]}$ igualmente, Merino plantea que "el hecho de que los cuidadores familiares se encuentren en la etapa adulta, significa tener que desempeñar diferentes roles, pues están en su máximo de productividad y desarrollo en su proyecto de vida. Esta condición ha determinado un factor vulnerable para vivir los efectos devastadores del estrés, experimentar más carga subjetiva que objetiva y, por consiguiente, ubicarse en un rango intermedio de manifestar afecciones psicológicas, al no sentir completamente control por las cosas que les suceden en la vida y estar alterada la satisfacción que les genera la misma".[22]

Las características de los menores, se relacionan con los hallazgos de Delucchi, donde la edad promedio de los niños era de 8,6 años y alta la incidencia de niños en diálisis peritoneal, la disminución en la edad de ingreso y el deterioro progresivo en su crecimiento requiere la presencia de un cuidador. Respecto al tratamiento, la apreciación de las cuidadoras se relaciona con lo encontrado por Tong, en este estudio se asocia con estrés, cansancio e impotencia.

Por otra parte, una enfermedad devastadora ocasiona en el cuidador una pérdida importante para su vida, que le lleva a un proceso de duelo; de acuerdo con Barreto es un proceso psicológico ante una pérdida importante en la vida ${ }^{[23]}$ y como en todo proceso es necesaria la utilización de medios para identificar oportunamente la situación, generar estrategias para contribuir con el afrontamiento y obtener resultados en su beneficio; corroborado por Andreu y Moreno "La carga que siente el cuidador está muy influida por las estrategias de afrontamiento al problema que tiene éste y de los recursos que se encuentran a su disposición. "[24]

Wong y cols. observaron que los padres de niños con cáncer durante el proceso de adaptación, utilizaron estrategias y superaron las dificultades llegando a identificar aspectos positivos de la experiencia de la enfermedad y establecer esperanza para el futuro; ${ }^{[25]}$ las cuidadoras de esta investigación no han alcanzado estos logros, puesto que en lugar de adaptarse a su realidad se resignaron, que sirve 
como estrategia para hacer frente al disconfort producido durante el duelo; a la resignación se puede llegar de dos formas, recibir un estímulo positivo lleva a una aceptación de la realidad que vive; mientras que si el estímulo es negativo se resigna en contra de su voluntad, considera su realidad como algo impuesto, al no tener otra opción.

Por otra parte, algunos aspectos que influyen en la conducta de los individuos pueden modificarse mediante las intervenciones externas empleadas, estas serán eficaces en la medida que sean brindadas por personal idóneo y de forma adecuada, es decir si se aborda al individuo teniendo cuenta su cultura, creencias y costumbres; Alameda y Barbero consideran que es necesario generar medios y estrategias para afrontar el sufrimiento y prevenir la aparición del duelo disfuncional, señalan que estas deben ser dadas de acuerdo a la cultura del individuo y por su la naturaleza constituyen una responsabilidad moral de los profesionales y las estructuras sanitarias. ${ }^{[26]}$ Además, Largo y cols., resaltan la importancia de que en el equipo de salud la labor del profesional de enfermeria, al considerar que el vínculo creado con los individuos les permite compartir sus situaciones, consideran que para ofrecer cuidados de calidad es necesario capacitarlos a nivel personal y profesional en el tema ${ }^{[27]}$.

Por otro lado, Krikorian y cols., en su estudio con cuidadores de niños con cáncer encontraron que el apoyo psicosocial, no fue percibido como un factor protector para disminuir su sufrimiento ${ }^{[28]}$ Contrario a esto, Li y cols., consideran que cuando el cuidador recibía apoyo emocional los niveles de ansiedad son menores y disminuyen la posibilidad de presentar morbilidades psicológicas. ${ }^{\text {[29] }}$ Igualmente, Romero y cols. Plantean que, los conflictos con otros miembros de la familia, la disminución de la autoestima y la carencia de una red de apoyo social pueden generar efectos negativos en la capacidad de afrontamiento efectivo del cuidador, exponiéndolo a sufrir de diversos problemas de salud, lo cual repercute negativamente en la calidad de vida del paciente, del cuidador $y$ de la farmilia en general. ${ }^{[30]}$ Los hallazgos guardan relación con lo concluido por Li y cols. y Romero y cols., teniendo en cuenta que el apoyo recibido del medio exterior influye en el duelo, cuando éste es eficaz, fomenta factores protectores en múltiples direcciones ya que aumenta el confort; así mismo, cuando las estrategia de gestión externas son ineficaces y no aumentan el confort, conduce a la resignación.

Ante las consideraciones anteriores, no se puede seguir ignorando los efectos que causa en él desempeñar el rol, éste al igual que el sujeto de cuidado necesita y tiene derecho a una atención integral, orientada a tratar y/o prevenir las complicaciones físicas, psicológicas, sociales y espirituales que se presentan. Blanco señala que en el proceso de atención al enfermo terminal también se debe involucrar al cuidador, ${ }^{[31]}$ esta inclusión aplica no solo a enfermos terminales sino también a enfermos crónicos, que ante la demanda de cuidados ven afectada su calidad de vida y sienten la necesidad de ser ayudados durante el desempeño del rol.

\section{Conclusiones}

Se concluye que las estrategias de gestión interna y externas eficaces se convierten en ineficaces si son antecedidas por la impotencia, ésta genera en el cuidador estrategias de gestión internas ineficaces como la culpa, que a su vez genera impotencia, es así como Stefani y otros, plantean una relación entre la culpa experimentada por los fracasos durante el cuidado y los consecuentes senti- mientos de impotencia ${ }^{[32]}$ que limita a las cuidadoras a implementar y realizar estrategias de gestión internas que permita hacer frente a la pérdida.

Finalmente, Enfermería para comprender la situación del cuidador debe profundizar en su realidad, ya que como lo menciona Montalvo son escasos los estudios sobre este tema, siendo necesario desde la disciplina comprender la persona y poder así asumir en el cuidado la totalidad del ser humano, con un trato digno y humanizado. ${ }^{[33}$

\section{Referencias}

1. Marriner A, Alligood M. Modelos y Teorías en Enfermería. $7^{a}$ ed. España: Elsevier; c2008. 850 p.

2. Pinto N. Bienestar espiritual de los cuidadores familiares de niños que viven enfermedad crónica. Investigación en Enfermería: Imagen y Desarrollo. 2007;9(1):20-35.

3. Barrera L, Pinto $N$, Sánchez B. Cuidando a los Cuidadores. Un programa de apoyo a familiares de personas con enfermedad crónica. Index Enferm [Internet]. 2006. Jun. [citado el 2 de Marzo de 2014], 15:52-53.

4. Santrock J. Psicología de la educación. México. Ed. Mc Graw- Hill; c 2002. 586 p.

5. Herrera F, Ramírez M, Roa, J, Herrera I. Tratamiento de las creencias motivacionales en contextos educativos pluriculturales. En: Revista Iberoamericana de Educación. [Internet] 2003 [citado el 2 de Marzo de 2013]. 37(2): 1-20.

6. Rodríguez R, Auné $S$, Vetere $G$. Regulación emociona en pacientes con trastornos de ansiedad: un estudio comparativo con una muestra comunitaria. En: Anu investig [Internet] 2012, Jun [citado el 12 de Dic. de 2014]; 19(1):273-280.

7. Pérez $P$ La culpa traumática y sus contextos: reflexiones sobre una psicología de las decisiones morales. Servicio Psiquiatría. Hospital La Paz. [Internet] [Citado el 2 de Marzo de 2013]

8. Vásquez $M$. La sobreprotección materna y su incidencia el bajo rendimiento escolar en niños y niñas comprendidos entre las edades de 6 a 12 años. [Internet]. Guatemala; 2007 Oct.

9. Estrada L. El ciclo vital de la familia. México. Ed. Grijalbo c2003.

10. Lopes $\vee$ A família e o processo de interiorização: trāa ${ }^{\mathrm{s}}$ exemplos. En: Arquivo brasileiro de psicologia. [Internet]. 1985 Jul-Sept. [Citado el 2 de Diciembre de 2013]. 37(3):105-111.

11. Nunes M, Rigotto D, Ferrari H, Marín F. Soporte social, familiar y autoconcepto: relación entre los constructos. En: Psicología desde el Caribe. [Internet]. 2012, Jun. [Citado el 10 de Diciembre de 2013]. 29(1):1-18.

12. Guía de apoyo para cuidadores. RAPPS (Red Argonesa de Proyectos de Promoción de Salud). Proyecto el cuidado del cuidador. [Internet]. Hospital San José Teruel. [Citado el 4 de Diciembre de 2013].

13. Sarason I, Levine H, Basham R, Sarason B. Assessing social support: the social support questionnaire. En: Journal of Personality and Social Psychology. [Internet].1983. [Citado el 2 de Marzo de 2014]; 44(1):127-139. 
14. Garrido A, Guadalupe L, Torres L, Ortega P Importancia de las expectativas de pareja en la dinámica familiar. En: Enseñanza e investigación en psicología. [Internet]. 2008 Jul- Dic.

15. Goodwin R, Cost P, Adonu J. Social support and its consequences: "positive" and "deficiency" values and their implications for support ans self- esteem. En: British Journal of Social Psychology. [Internet]. 2004. [Citado el 12 de Diciembre de 2013]; 43: pp. 1-10.

16. Diagnósticos enfermeros: definición y clasificación 2005 2006. NANDA International. North American Nursing Diagnosis Association. Elsevier. 2005.

17. Pinto Afanador N, Sánchez Herrera B. El reto de los cuidadores: familiares de personas en situación crónica de enfermedad. Bogotá: Unibiblos, 2000.

18. Andreu L, Gruart P, Tamar L. Visión enfermera de las necesidades psicosociales de los cuidadores de personas en tratamiento con Diálisis Peritoneal. En: Rev Soc Enferm Nefrol [Internet]. 2007. [citado el 1 de Diciembre de 2013]; 10(1):47-52.

19. Blanco D. Habilidad de cuidado de los cuidadores principales de niños en situación de enfermedad crónica que consultan en la Clínica Colsanitas. En: Avances en Enfermería. 2007;25(1):19-32.

20. Tong A, Lowe A, Sainsbury P, Craig J. Parental perspectives on caring for a child with chronic kidney disease: an in-depth interview study. En: Child: care, health and development. [Internet]. 2010, Nov. [Citado el 5 de Diciembre de 2013]. 36(4):549-557.

21. Montalvo A, Flórez I. Características de los cuidadores de personas en situación de cronicidad. Cartagena (Colombia). Un estudio comparativo. En: Salud Uninorte. 2008;24(2):181-190.

22. Merino S. Calidad de vida de los cuidadores familiares que cuidan niños en situaciones de enfermedad crónica. En: Avances en Enfermería. 2004;22(1):39-46.

23. Barreto P, Yi P, Soler C. Predictores de duelo complicado. En: Psicooncología. [Internet] 2008.
24. Andreu L, Moreno M. Perfil y realidad social de los cuidadores principales de pacientes dependientes tratados con hemodiálisis. En: Nursing. [Internet] 2009. [Citado el 20 de Febrero de 2014]; 27(4):54-65.

25. Wong MI.The qualitative experience of Chinese parents with children diagnosed of cancer. En: Journal of Clinical Nursing. [Internet]. 2006, Jun.

26. Aladema A, Barbero J. El duelo en padres del niño oncológico. En: Psicooncología. [Internet]. 2009. [Citado el 25 de Noviembre de 2013]; 6(2-3):485-498.

27. Largo F, Camacho R, López S, Domínguez M, Aragón $M$, Correa L. Afrontamiento eficaz del duelo: el proceso MAR. En: Metas de enfermería. [Internet]. 2003. [citado el 29 de Noviembre de 2013]; 6(61):12-16.

28. Krikorian D, Vélez M, González O, Palacio C, Vargas J. La experiencia de sufrimiento en cuidadores principales de pacientes con dolor oncológico y no oncológico. En: Avances en enfermería. 2010;28(1):13-20.

29. Li R, Cooper C, Bradley J, Shulman A, Livingston Gill. Coping strategies and psychological morbidity in family carers of people with dementia: A systematic review and meta-analysis. En: Journal of Affective Disorders. [Internet]. 2012.

30. Romero E, Macausland Y, Solórzano L. El cuidador familiar del paciente renal y su calidad de vida, Cartagena (Colombia). En: Salud Uninorte. 2014;30(2):146-157.

31. Blanco L, Flores L, Rocafort G. Perfil del cuidador principal del enfermo en situación terminal y análisis del riesgo de desarrollar duelo patológico. Medicina paliativa. [Internet]. 2007. [Citado el 28 de Noviembre de 2013]; 14(3) 164-168.

32. Stefani D, Seidmann S, Pano C, Acrich L, Bail V Los cuidadores familiares de enfermos crónicos: sentimientos de soledad, aislamiento social y estilos de afrontamiento. Revista Latinoamericana de Psicología. [Internet]. 2003. [Citado el 27 de Noviembre de 2013]; 35(1):55-65.

33. Montalvo A, Cabrera B, Quiñones S. Enfermedad crónica y sufrimiento: revisión de literatura. En: Aquichan. $2012 ; 12(2): 134-143$ 